\title{
Effect of fexofenadine/pseudoephedrine combination tablet on nasal obstruction in patients with allergic rhinitis using rhinomanometry: A randomized controlled trial
}

\author{
Yosuke Nakamura, ${ }^{1}$ Yuko Yokoyama, ${ }^{1}$ Satoshi Koyama, ${ }_{1}$ Kazunori Fujiwara, ${ }^{1}$ Motoki Nakamori, ${ }^{1}$ \\ Taihei Fujii, ${ }^{2}$ Tadao Enomoto, ${ }^{3}$ Hiromi Takeuchi ${ }^{1}$
}

\begin{abstract}
Background: A fexofenadine/pseudoephedrine combination tablet $(\mathrm{F} / \mathrm{P})$ is an optimal product for nasal obstruction. It contains fexofenadine hydrochloride, a histamine $\mathrm{H}_{1}$-receptor antagonist for sneezing and rhinorrhea and pseudoephedrine hydrochloride, an a-adrenergic agonist. The effect of an antihistamine-decongestant on nasal obstruction has been demonstrated in previous studies, but onset of action and efficacy data on nasal obstruction are limited.
\end{abstract}

Objective: We estimated the efficacy of F/P on nasal obstruction in patients with house dust mite-induced allergic rhinitis (AR) versus fexofenadine (F) using objective methods.

Methods: In this single-center, single-dose, prospective, randomized, parallel-group study, 24 adult patients with a history of at least 2 years of AR and nasal obstruction were randomized to receive F/P or F. The effect on nasal obstruction was evaluated using nasal airflow and visual analog scale (VAS) score measured at 30-minute intervals before and for 8 hours after dosing. The primary end point was onset of action, based on a comparison of absolute change from baseline in nasal airflow between F/P and F. The protocol was registered in a clinical trial registry as UMIN 000041845.

Results: The onset of action for F/P was 30 minutes based on nasal airflow and 60 minutes based on VAS. F/P maintained a significant beneficial effect after onset of effect, while $\mathrm{F}$ showed no significant change during the test period.

Conclusions: We found F/P had a clear effect on nasal obstruction associated with perennial AR when compared with F. There was a time lag in nasal airflow improvement and nasal obstruction relief.

Key words: Allergic rhinitis, Antihistamine, Nasal obstruction, Rhinomanometry, Visual analog scale

\footnotetext{
From:

Division of Otolaryngology, Head and Neck Surgery, Faculty of Medicine Tottori University,

Division of Head, Neck, and Thyroid Surgery Center and Otorhinolaryngology, Kusatsu General Hospital

NPO Japan Health Promotion Supporting Network
}

\section{Introduction}

Allergic rhinitis (AR) affects an estimated $10 \%$ to $30 \%$ of the population worldwide $e^{1,2,3}$ and is associated with considerable cost and comorbidity. ${ }^{4,5}$ In some parts of the world, the prevalence of AR in children reaches $40 \%$ or more. ${ }^{6,7}$ In Japan, the prevalence of AR increased from $29.8 \%$ to $39.4 \%$ between 1998 and $2008 .^{8}$ AR has attracted increasing attention worldwide due to its impact on quality of life and diseases such as asthma, as documented in the Allergic Rhinitis and its Impact on Asthma (ARIA) document. ${ }^{9}$

\author{
Corresponding author: \\ Yosuke Nakamura \\ Division of Otolaryngology, Head and Neck Surgery, \\ Faculty of Medicine, Tottori University, \\ 36-1 Nishicho, Yonago, Tottori 683-8504, Japan \\ E-mail: yosuken@tottori-u.ac.jp
}

Antihistamines have been used for many years as firstline therapy for the treatment of AR. It remains an important treatment option. ${ }^{6,8,9}$ Antihistamines block the $\mathrm{H}_{1}$ receptor. They have been shown to reduce rhinorrhea, pruritus and sneezing in studies. ${ }^{10,11}$ Fexofenadine $(\mathrm{F})$ is one of the most widely used second-generation antihistamines ${ }^{12}$ that is also popular in Japan. Although there are a few studies illustrating that newer antihistamines reduce nasal obstruction, those drugs are generally shown to be less effective against nasal obstruction compared to other symptoms such as sneezing and itching. ${ }^{13-16}$ 
When combined with a-decongestants, antihistamines have been shown to control a wide range of rhinitis symptoms not only in runny nose and sneezing, but also in nasal obstruction..$^{8,17,18}$ Currently, pseudoephedrine $(\mathrm{P})$ is a widely used nasal decongestant. It is available in numerous over the-counter products. ${ }^{19}$ A few studies have verified the onset of action of non-sedating antihistamine-decongestant combination products. ${ }^{20-22}$ So far, there has been no reports that have objectively showed onset of action for the F and P combination.

Nasal obstruction can be measured in the laboratory with rhinomanometry (RM). Anterior RM is a well-defined laboratory tool that measures function in terms of nasal resistance or nasal airflow, which have been validated as sensitive measures for evaluating rhinitis. ${ }^{23,24}$ Few reports have evaluated the effects of drugs on nasal obstruction over time in detail.

The purpose of this study was to evaluate the onset of action and efficacy against nasal obstruction of a single dose of combination of F hydrochloride (HCI) $60 \mathrm{mg}$ and P HCI 120 mg versus $\mathrm{F}$ in patients with house dust mite-induced AR using RM. We investigated the time difference between subjective and objective evaluation onset of action.

\section{Methods \\ Patients}

We recruited 24 patients (13 men and 11 women) with perennial AR from the Division of Otolaryngology, Head and Neck Surgery at Tottori University Hospital in Japan between January 2015 and December 2016. The diagnosis of perennial AR was based on the ARIA guidelines ${ }^{9}$ and Japanese guidelines for AR. ${ }^{8}$

The study protocol (No. 2232) was approved by the Committee on Human Research of Tottori University in accordance with the Declaration of Helsinki. All patients were informed of our treatment protocols and provided written consent before participation. The protocol was registered in a clinical trial registry as UMIN 000041845.

Inclusion criteria included (1) a history of at least two of the following symptoms: sneezing, watery rhinorrhea or nasal obstruction, for at least 2 years, and (2) house dust mite allergy based on skin reactions or serum allergen-specific IgE antibody measurements.

Exclusion criteria were the existence of major structural nasal abnormalities, upper respiratory tract infection during the previous 4 weeks, allergen-specific immunotherapy, steroid or antileukotriene therapy in the last 4 weeks, antihistamine use in the last 4 days, pregnancy, or lactation. None of the study participants were free of nasal obstruction or had undergone any previous sinonasal surgery. Similarly, patients with hypertension, severe asthma, and a history of known drug hypersensitivity and adverse events, or intolerance to $\mathrm{F}$ or F/P were also excluded.

Skin-prick test were performed with common aeroallergens such as house dust mite. We used a negative control to determine 15 minutes after injection. The reaction was regarded as positive if the mean wheal diameter was at least 5 $\mathrm{mm}$ or flare diameter was at least $15 \mathrm{~mm}$ based on Japanese clinical practice guidelines of AR. Serum specific IgE levels greater than class 2 were considered positive. No patient had other inhalant allergies that could have affected the symptoms at the time of the study.

\section{Study design}

We conducted a single-center, single-dose, prospective, randomized, parallel-group study. Patients who met the inclusion criteria participated in the study. Patients arrived at the lab in the morning for the first set of measurements.

Patients were assigned to one of two study groups, F/P or $\mathrm{F}$, based on simple randomization in a $1: 1$ ratio with the use of a computerized system for generating random numbers. Patients in the F/P group took fexofenadine $60 \mathrm{mg}$ and pseudoephedrine $120 \mathrm{mg}$ and patients in the $\mathrm{F}$ group took fexofenadine $60 \mathrm{mg}$. Patients were blinded to their anti-allergy medication group assignment. Patients in the F/P group were instructed to take the drug between meals while patients in the $\mathrm{F}$ group were instructed to take the drug after meals. Measurement started at 9-12 am. During the 480-minute test period, patients were given a private room and were free to spend time in the room and return to the nearby lab at each time point for data collection. Alcohol, coffee, physical activities, changes of temperature and hot meals or beverages were avoided during the study.

\section{Efficacy assessments}

The effect of each medication on nasal obstruction was evaluated based on nasal airflow and VAS score. Nasal airflow was measured at 30 minute intervals before and for $480 \mathrm{~min}$ utes after dosing. VAS score was recorded at the same time. Anterior RM was used to assess nasal patency (A1 Executive Acoustic Rhinometer GM Instruments, Ltd., Kilwinning, UK). Nasal airflow was calculated as the sum of left and right RM measurements at 100 Pascal, as previously reported by Ohki et al. ${ }^{23,25}$ Nasal airway resistance was defined as the reciprocal of nasal airflow. Nasal obstruction was evaluated on a VAS, with 0 representing no problems and 10 representing intolerable conditions.

The primary end point was onset of action, based on a comparison of absolute change from baseline in nasal airflow between F/P and F. Onset of action was defined as the first time between 30 minutes and 480 minutes after dosing that a consistent, statistically significant change in nasal airflow was observed for F/P relative to F. Secondary end points included the absolute change in VAS score. Onset of action was defined in the same way for VAS score as for nasal airflow. In addition, we examined the time at which peak improvement in nasal airflow and VAS occured.

\section{Statistical analysis}

The efficacy assessment was based on the per protocol set (PPS) population, which consisted of all patients who were randomized to treatment and took a single dose of the study medication according to the protocol.

Based on statistical reference to pilot studies, ${ }^{26}$ we have defined 24 as an appropriate sample size because there was no similar study so far.

Statistical analyses were performed using SPSS for Windows, version 25 (IBM Corp. Armonk, NY, USA). Baseline characteristics were compared between the two groups using 
the independent sample $t$-test and the chi-square test. The paired $t$-test and Wilcoxon signed-rank test were used for intragroup comparisons. Repeated measures analysis of variance was employed to compare treatment results between two arms of trial. In all tests, $p$ value $<0.05$ was considered as statistically significant.

\section{Results}

As shown in Figure 1, the 24 eligible participants were randomly allocated into two groups: F/P group $(\mathrm{n}=12)$ and F group $(n=12)$. Therefore, the PPS included 24 participants who took their assigned study medication. Demographic and baseline characteristics were similar across the two treatment groups (Table 1).

The onset of action for $\mathrm{F} / \mathrm{P}$, as measured by increase in $\mathrm{ab}$ solute nasal airflow, was 30 minutes ( $p<0.001$ vs. F) (Figure 2 ). Increases in absolute nasal airflow were significantly greater with $\mathrm{F} / \mathrm{P}$ than with $\mathrm{F}$ at all subsequent time points up to 480 minutes after dosing (all $p<0.001$ ). Administration of $\mathrm{F} / \mathrm{P}$ resulted in a marked increase in nasal airflow between 0 and 30 minutes (Figure 2). Maximal increase in nasal airflow occured at 180 minutes after F/P administration (Figure 2).

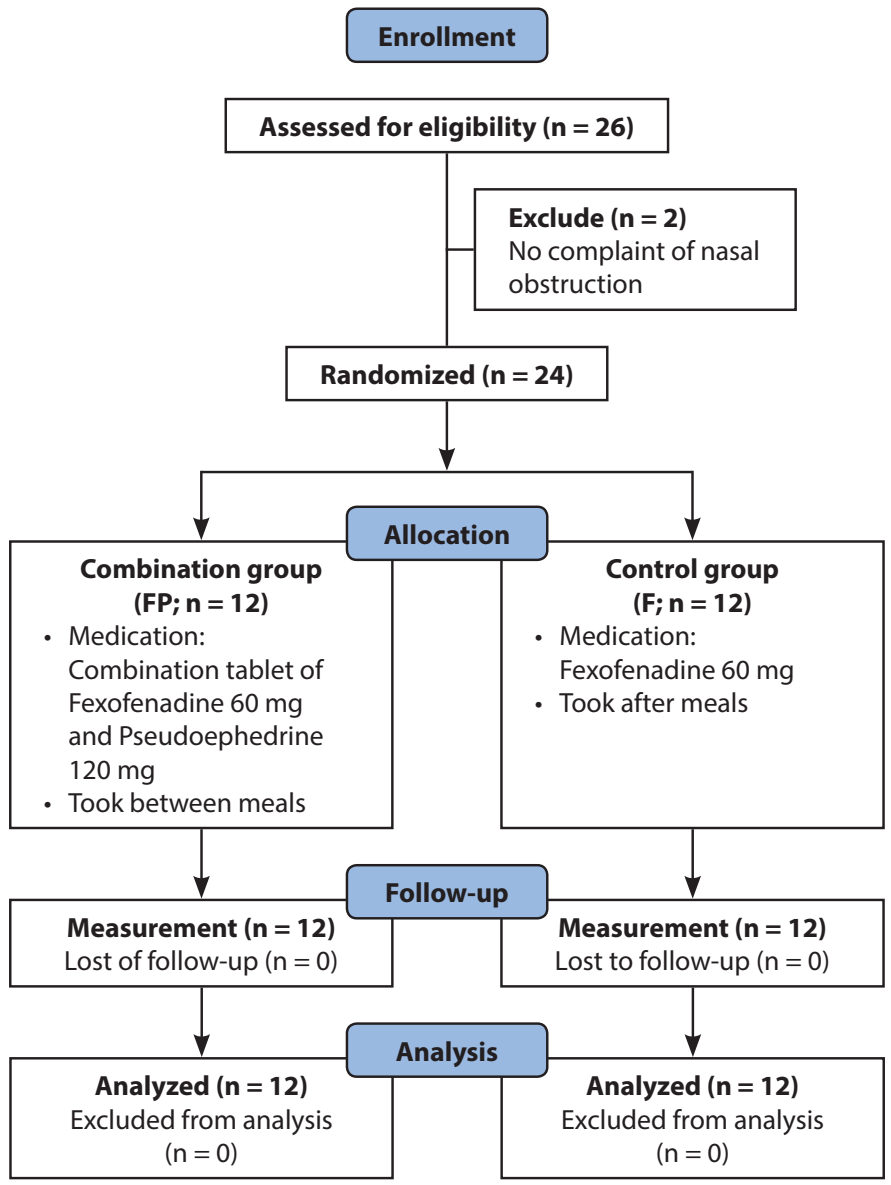

Figure 1. Diagram for subject enrollment, allocation, follow-up and analysis.
For F/P, the onset of action, based on decrease in absolute VAS score was 60 minutes ( $p<0.001$ vs. F) (Figure 3). Administration of $\mathrm{F} / \mathrm{P}$ resulted in a marked decrease in absolute VAS score between 30 and 60 minutes (Figure 3). Decreases in absolute VAS score were significantly greater with $\mathrm{F} / \mathrm{P}$ than with $\mathrm{F}$ at each time point from 60 minutes after dosing to the end of the study (all $p<0.001$ ) (Figure 3). Furthermore, the maximal decrease in absolute VAS score occurred at $330 \mathrm{~min}$ utes after F/P administration (Figure 3).

There was a significant change in nasal airflow and VAS score with $\mathrm{F} / \mathrm{P}$ at 60,180 , and 330 minutes (all $p<0.05$ vs. baseline). With F, nasal airflow and VAS score were not significantly different from baseline over the entire 480-minute period (Figure 2) (Figure 3). Nasal airflow showed a tendency to increase slightly throughout the period with fluctuation (Figure 2). Table 2 shows the amount of change in nasal airflow at representative time points. In $\mathrm{F} / \mathrm{P}$ total nasal resistance at each time point was $0.33,0.27,0.28,0.25,0.23,0.26$, and 0.24 , respectively.

Table 1. Demographics and Baseline Characteristics.

\begin{tabular}{|c|c|c|c|}
\hline Factors & $\begin{array}{c}\text { F/P } \\
(n=12)\end{array}$ & $\begin{array}{c}F \\
(n=12)\end{array}$ & $p$ value \\
\hline Gender (female, \%) & $5(41.7)$ & $6(50.0)$ & 0.682 \\
\hline Age (mean $\pm S E)$ (year) & $30.4 \pm 2.2$ & $31.8 \pm 3.3$ & 0.745 \\
\hline Sensitization to HDM & & & 0.584 \\
\hline Specific IgE (count, \%) & $11(91.7)$ & $9(75.0)$ & \\
\hline Class 2-3 (count) & 10 & 7 & \\
\hline Class 4-6 (count) & 1 & 2 & \\
\hline Skin prick test (count, \%) & $1(8.3)$ & $3(25.0)$ & \\
\hline$+($ count $)$ & 1 & 3 & \\
\hline Grade & & & 0.701 \\
\hline Mild (count, \%) & $5(41.7)$ & $4(33.3)$ & \\
\hline Moderate (count, \%) & $4(33.3)$ & $6(50.0)$ & \\
\hline Severe (count, \%) & $3(25.0)$ & $2(16.7)$ & \\
\hline Symptom score (mean \pm SE) & $3.8 \pm 0.5$ & $3.9 \pm 0.6$ & 0.822 \\
\hline $\begin{array}{l}\text { Nasal airflow (mean } \pm \text { SE) } \\
\left(\mathrm{cm}^{3} / \mathrm{s}\right)\end{array}$ & $341.0 \pm 27.9$ & $316.2 \pm 23.4$ & 0.500 \\
\hline VAS score (mean \pm SE) & $4.9 \pm 0.7$ & $4.3 \pm 0.4$ & 0.472 \\
\hline Allergic diseases & & & 0.875 \\
\hline BA (count, \%) & $2(16.7)$ & $2(16.7)$ & \\
\hline AD (count, \%) & $3(25.0)$ & $2(16.7)$ & \\
\hline
\end{tabular}

F/P, Fexofenadine and Pseudoephedrine combination tablet; F, Fexofenadine; HDM, house dust mite; + showing positive.

$\mathrm{AD}$, atopic dermatitis; $\mathrm{BA}$, bronchial asthma; SE, standard error. 


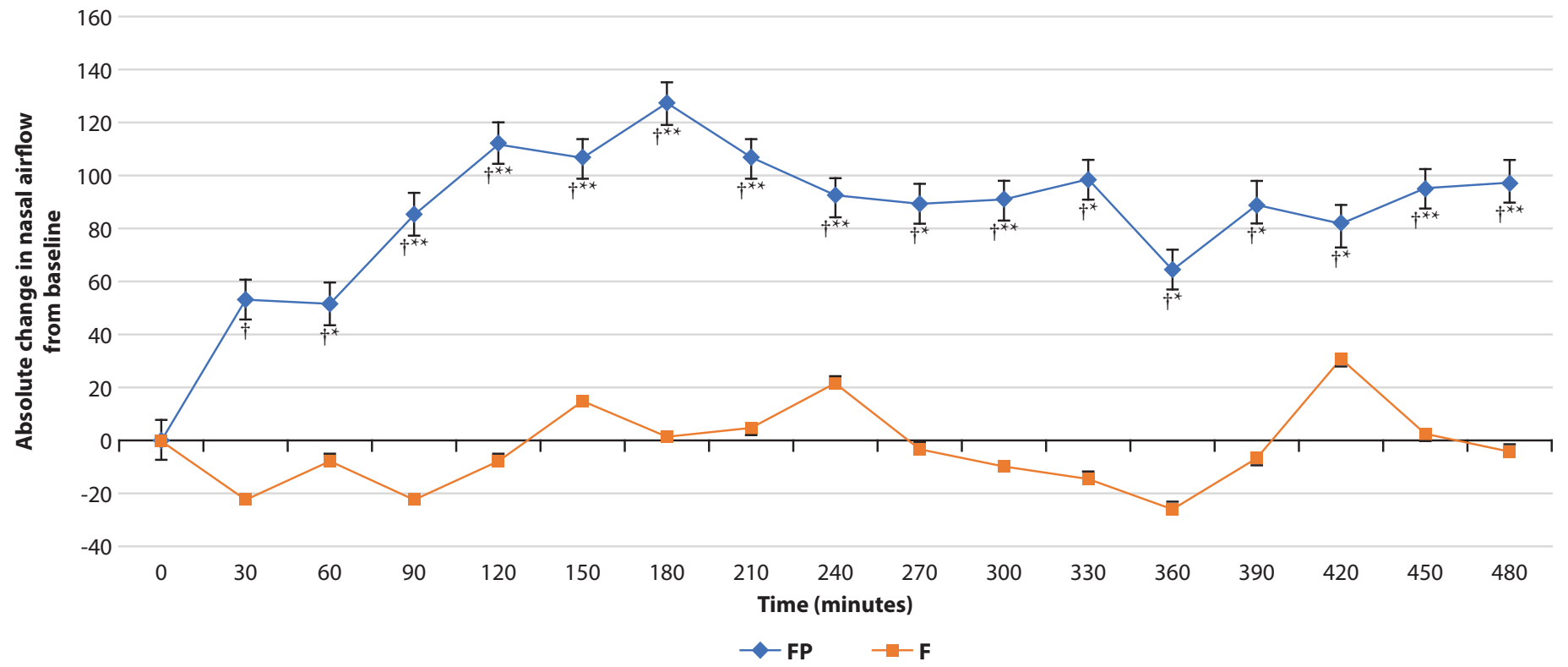

Figure 2. Mean change in nasal airflow from baseline for the $F / P$ and $F$ groups.

${ }^{\dagger} P<0.001$ vs. F. ${ }^{*} P<0.05,{ }^{*} P<0.01$ vs. baseline. Error bars indicate standard error.

F/P, Fexofenadine and Pseudoephedrine combination tablet; F, Fexofenadine.

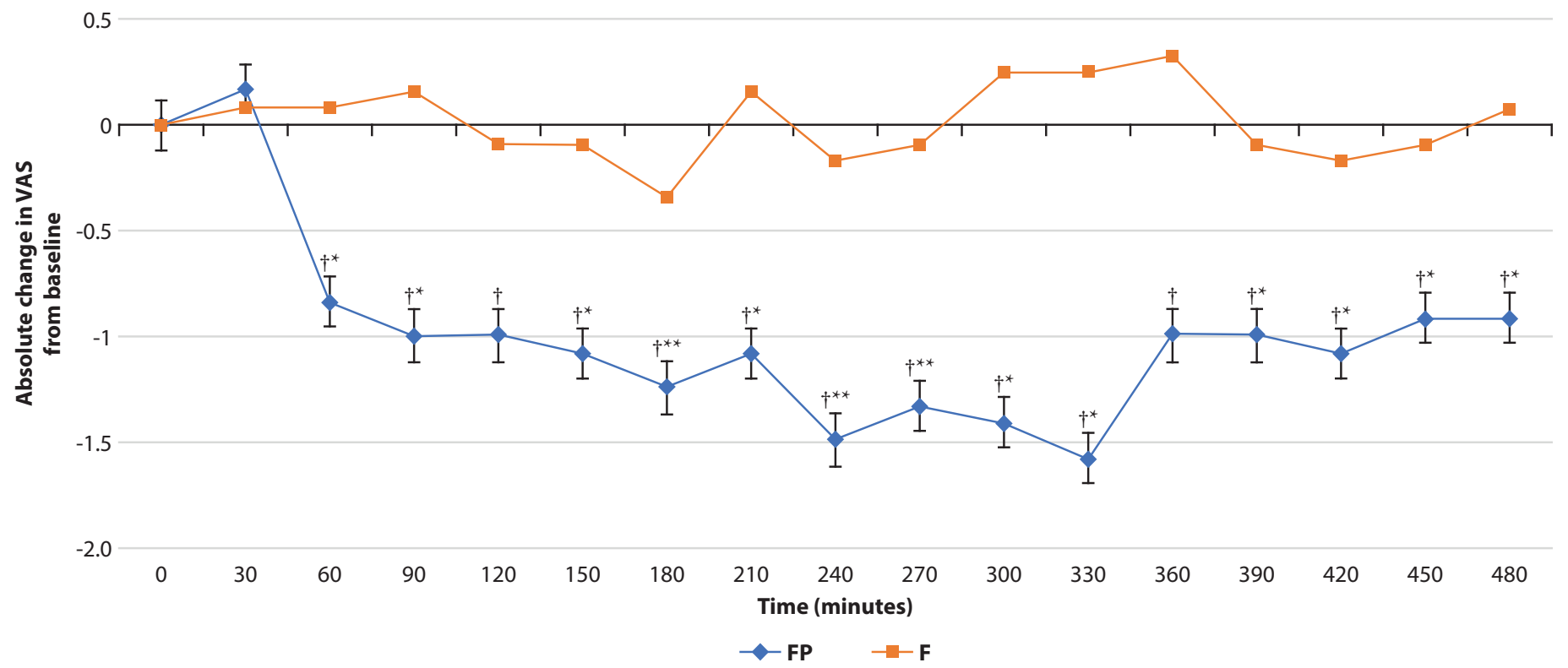

Figure 3. Mean change in VAS from baseline for F/P and F groups. Absolute VAS change.

${ }^{\dagger} P<0.001$ vs. $\mathrm{F} .{ }^{*} P<0.05,{ }^{*} P<0.01$ vs baseline. Error bars indicate standard error.

F/P, Fexofenadine and Pseudoephedrine combination tablet; F, Fexofenadine.

Table 2. Changes in nasal air permeability due to $\mathrm{F} / \mathrm{P}$ and $\mathrm{F}$.

\begin{tabular}{l}
\multicolumn{1}{c}{ Time (minutes) } \\
F/P
\end{tabular}


Table 2. (Continued)

\begin{tabular}{|c|c|c|c|c|c|c|}
\hline Time (minutes) & 30 & 60 & 90 & 180 & 330 & 480 \\
\hline \multicolumn{7}{|l|}{ F/P (Continued) } \\
\hline \multicolumn{7}{|l|}{ VAS } \\
\hline Mean changes & 0.2 & $-0.8^{*}$ & $-1.0^{*}$ & $-1.3^{\star *}$ & $-1.6^{*}$ & $-0.9^{*}$ \\
\hline SE & 0.2 & 0.3 & 0.4 & 0.3 & 0.5 & 0.4 \\
\hline $95 \% \mathrm{CI}$ & $-0.4,0.7$ & $-1.5,-0.2$ & $-1.9,-0.1$ & $-1.9,-0.6$ & $-2.6,-0.5$ & $-1.8,0$ \\
\hline \multicolumn{7}{|l|}{$\mathbf{F}$} \\
\hline \multicolumn{7}{|l|}{ Nasal airflow } \\
\hline Mean changes $\left(\mathrm{cm}^{3} / \mathrm{s}\right)$ & -23.1 & -7.6 & -22.7 & 0.8 & -14.5 & -4.1 \\
\hline $\mathrm{SE}\left(\mathrm{cm}^{3} / \mathrm{s}\right)$ & 11.8 & 18.0 & 18.4 & 17.5 & 18.9 & 25.6 \\
\hline $95 \% \mathrm{CI}\left(\mathrm{cm}^{3} / \mathrm{s}\right)$ & $-49.2,2.9$ & $-47.4,32.2$ & $-63.4,17.9$ & $-37.7,39.2$ & $-56.2,27.2$ & $-60.5,52.2$ \\
\hline \multicolumn{7}{|l|}{ VAS } \\
\hline Mean changes & 0.1 & 0.1 & 0.2 & -0.3 & 0.3 & 0.1 \\
\hline SE & 0.2 & 0.2 & 0.3 & 0.5 & 0.4 & 0.6 \\
\hline $95 \%$ CI & $-0.4,0.6$ & $-0.3,0.5$ & $-0.6,0.9$ & $-1.4,0.7$ & $-0.7,1.2$ & $-1.3,1.5$ \\
\hline
\end{tabular}

F/P, Fexofenadine and Pseudoephedrine combination tablet; CI, confidence interval (95\%); SE, standard error. ${ }^{*} P<0.05,{ }^{*} P<0.01$ vs baseline.

\section{Discussion}

This study focused on nasal obstruction in AR affecting quality of life (QOL). Using objective (RM) and subjective (VAS score) indicators, we sought to examine the onset of action and efficacy of a single dose of F HCl $60 \mathrm{mg} / \mathrm{P} \mathrm{HCl} 120$ mg compared with $\mathrm{F} \mathrm{HCl} 60 \mathrm{mg}$ in patients with house dust mite-induced AR. We observed an immediate effect of improved nasal airflow after $\mathrm{F} / \mathrm{P}$ administration and a sustained effect during the 480 -minutes test period.

In this study, RM analysis showed that $\mathrm{F} / \mathrm{P}$ significantly and rapidly increased absolute nasal airflow with onset of action at 30 minutes. This is consistent with earlier studies employing RM in which the action of $\mathrm{P}$ was apparent at 30 to 60 minutes after dosing. ${ }^{27-29}$ The previous study showed that the decrease in nasal obstruction in subjects treated with $\mathrm{P}$ alone or in combination with $\mathrm{F}$ is attributed to the effect of the decongestant on the nasal vasculature. ${ }^{27}$ This study was conducted using RM to determine whether F/P makes a distinct contribution to the relief of nasal obstruction symptoms of AR. $\mathrm{F} / \mathrm{P}$ was found to have an onset of 30 minutes. The rapid effect of F/P in the study in decreasing nasal obstruction may be due to the effect of P. This is the first report to objectively evaluate the effect and examine the onset of action of F/P using RM.

Previous research on F/P was primarily based on subjective evaluation. For example, based on symptom scores, onset of decreased nasal obstruction occurred by 45 minutes. ${ }^{21,22}$ When VAS score was used to evaluate nasal obstruction in this study, the onset time was 60 minutes. The slightly different times to onset could be due to different measurement intervals or different control agents. In the present study, the active drug $\mathrm{F}$ was used as a control, whereas previous reports mentioned a large placebo effect. ${ }^{30}$ In an environmental exposure unit study, F did not significantly decrease nasal obstruction compared with placebo throughout the 5-hour exposure period. ${ }^{31}$ Although the study design was different, F showed no significant change in VAS score compared to baseline during the 8-hour study period in this study. The effects of antihistamines on nasal obstruction have traditionally been obscure. ${ }^{16}$ Based on results discussed above, the effect of antihistamines such as $\mathrm{F}$ on nasal obstruction may be poor. By adding an objective evaluation, it became clear that $\mathrm{F}$ had a poor effect on obstruction but F/P had an effect on obstruction.

It is unclear why the onset of action in the objective evaluation was 30 minutes earlier than onset of action in the subjective evaluation in this study. Considering that the sensation of nasal obstruction is based on perceived airflow in the nasal cavity, there may be a slight time lag between changes in nasal airflow and VAS score. Nasal air flow is perceived indirectly by cold receptors in the nasal cavity. ${ }^{32}$ These receptors are supplied by branches of the trigeminal nerve, ${ }^{33}$ and perception of nasal airflow has been reported to be unrelated to other organs. ${ }^{34}$ As a result of measuring both subjective and objective evaluations at the same time, the time when nasal airflow and VAS was the most different from baseline may have been different for the same reason. These results are in agreement with the results of a study of $\mathrm{P}$ with a similar approach. ${ }^{29}$ Since previous studies have only evaluated the effect of drugs on nasal obstruction either subjectively or objectively, the effect on the perception of nasal obstruction was speculative. It was important to be able to evaluate the time lag between the two evaluations methods. 
By simultaneously performing subjective and objective, it was possible to approach the elucidation of the mechanism.

There are some limitations to this study. One limitation is the small number of samples. Second, the design of this study differed from the design of an environmental exposure unit study or park study. We focused on the effects of AR on nasal obstruction by including patients' with nasal obstruction. It would be difficult to use these other designs because the use of RM is needed to assess nasal obstruction.

In conclusion, compared with $\mathrm{F}$, a single dose of $\mathrm{F} / \mathrm{P}$ was effective in treating nasal obstruction in perennial AR with an onset of action of 30 minutes. Based on the VAS score for nasal obstruction, the onset of action was 60 minutes. F/P may be suitable for on-demand use due to its fast onset of action. There was a slight time lag between onset in the two evaluations, but elucidation of the mechanism of action is a topic for further study.

\section{Acknowledgments}

We gratefully acknowledge the work of past and present members of our colleague.

\section{Conflict of interest}

None.

\section{Author Contributions}

- YN and HT designed the study.

- YY, SK and MN contributed to data collection.

- TF was involved in creating the allocation table.

- YN, KF and TE performed the statistical analysis and interpretation of the results.

- YN wrote the manuscript.

- YN, YY, SK, KF, MN and TF edited.

- HT supervised. All authors read and approved the final manuscript

\section{References}

1. Asher MI, Montefort S, Björkstén B, Lai CK, Strachan DP, Weiland SK, et al. Worldwide time trends in the prevalence of symptoms of asthma, allergic rhinoconjunctivitis, and eczema in childhood: ISAAC Phases One and Three repeat multicountry cross-sectional surveys. Lancet. 2006;368: 733-43.

2. Brozek JL, Bousquet J, Baena-Cagnani CE, Bonini S, Canonica GW, Casale TB, et al. Allergic Rhinitis and its Impact on Asthma (ARIA) guidelines: 2010 revision. J Allergy Clin Immunol. 2010;126:466-76.

3. Watts AM, West NP, Smith PK, Cripps AW, Cox AJ. Probiotics and Allergic Rhinitis: A Simon Two-Stage Design to Determine Effectiveness. J Altern Complement Med. 2016;22:1007-12.

4. Incorvaia C, Cavaliere C, Frati F, Masieri S. Allergic rhinitis. J Biol Regul Homeost Agents. 2018;32:61-6.

5. Meltzer EO, Bukstein DA. The economic impact of allergic rhinitis and current guidelines for treatment. Ann Allergy Asthma Immunol. 2011;106: S12-6.

6. Seidman MD, Gurgel RK, Lin SY, Schwartz SR, Baroody FM, Bonner JR, et al. Clinical practice guideline: Allergic rhinitis. Otolaryngol Head Neck Surg. 2015;152:S1-43.

7. Wise SK, Lin SY, Toskala E, Orlandi RR, Akdis CA, Alt JA, et al International Consensus Statement on Allergy and Rhinology: Allergic Rhinitis. Int Forum Allergy Rhinol. 2018;8:108-352.

8. Okubo K, Kurono Y, Ichimura K, Enomoto T, Okamoto Y, Kawauchi H, et al. Japanese guidelines for allergic rhinitis 2020. Allergol Int. 2020;69(3): 331-45.
9. Bousquet J, Schünemann HJ, Samolinski B, Demoly P, Baena-Cagnani CE, Bachert C, et al. Allergic Rhinitis and its Impact on Asthma (ARIA): achievements in 10 years and future needs. J Allergy Clin Immunol. 2012;130:1049-62.

10. Meltzer EO, Weiler JM, Widlitz MD. Comparative outdoor study of the efficacy, onset and duration of action, and safety of cetirizine, loratadine, and placebo for seasonal allergic rhinitis. J Allergy Clin Immunol. 1996;97: 617-26.

11. Day JH, Briscoe MP, Welsh A, Smith JN, Clark A, Ellis AK, et al. Onset of action, efficacy, and safety of a single dose of fexofenadine hydrochloride for ragweed allergy using an environmental exposure unit. Ann Allergy Asthma Immunol. 1997;79:533-40.

12. Huang CZ, Jiang ZH, Wang J, Luo Y, Peng H. Antihistamine effects and safety of fexofenadine: a systematic review and Meta-analysis of randomized controlled trials. BMC Pharmacol Toxicol. 2019;20:72.

13. Nayak AS, Schenkel E. Desloratadine reduces nasal congestion in patients with intermittent allergic rhinitis. Allergy. 2001;56:1077-80.

14. Horak F, Stübner UP, Zieglmayer R, Harris AG. Effect of desloratadine versus placebo on nasal airflow and subjective measures of nasal obstruction in subjects with grass pollen-induced allergic rhinitis in an allergen-exposure unit. J Allergy Clin Immunol. 2002;109:956-61.

15. Simons FE, Simons KJ. Histamine and $\mathrm{H}_{1}$-antihistamines: celebrating a century of progress. J Allergy Clin Immunol. 2011;128:1139-50.e4.

16. Dávila I, Sastre J, Mullol J, Montoro J, Jáuregui I, Ferrer M, et al. Effect of bilastine upon nasal obstruction. J Investig Allergol Clin Immunol. 2011;21 Suppl 3:2-8.

17. Sussman GL, Mason J, Compton D, Stewart J, Ricard N. The efficacy and safety of fexofenadine $\mathrm{HCl}$ and pseudoephedrine, alone and in combination, in seasonal allergic rhinitis. J Allergy Clin Immunol. 1999; 104:100-6.

18. Badorrek P. A combination of cetirizine and pseudoephedrine has therapeutic benefits when compared to single drug treatment in allergic rhinitis. Int J Clin Pharmacol Ther. 2009;

19. Fashner J, Ericson K, Werner S. Treatment of the common cold in children and adults. Am Fam Physician. 2012;86:153-9.

20. Georgitis JW, Meltzer EO, Kaliner M, Weiler J, Berkowitz R. Onset-of-action for antihistamine and decongestant combinations during an outdoor challenge. Ann Allergy Asthma Immunol. 2000;84:451-9.

21. Berkowitz RB, Woodworth GG, Lutz C, Weiler K, Weiler J, Moss $\mathrm{M}$, et al. Onset of action, efficacy, and safety of fexofenadine $60 \mathrm{mg} /$ pseudoephedrine $120 \mathrm{mg}$ versus placebo in the Atlanta allergen exposure unit. Ann Allergy Asthma Immunol. 2002;89:38-45.

22. Berkowitz RB, McCafferty F, Lutz C, Bazelmans D, Godfrey P, Meeves S, et al. Onset of action of fexofenadine hydrochloride $60 \mathrm{mg} /$ pseudoephedrine hydrochloride $120 \mathrm{mg}$ in subjects aged 12 years with moderate to severe seasonal allergic rhinitis: a pooled analysis of two single-dose, randomized, double-blind, placebo-controlled allergen exposure unit studies. Clin Ther. 2006;28:1658-69.

23. Kim SJ, Choi JH, Kim EJ, Lee SK, Lee SH, Jun YJ, et al. A Prospective Population-based Study of Total Nasal Resistance in Korean Subjects. Clin Exp Otorhinolaryngol. 2012;5:39-43.

24. Passàli D, Salerni L, Passàli GC, Passàli FM, Bellussi L. Nasal decongestants in the treatment of chronic nasal obstruction: efficacy and safety of use. Expert Opin Drug Saf. 2006;5:783-90.

25. Ohki M, Hasegawa M. Studies of transnasal pressure and airflow values in a Japanese population. Rhinology. 1986;24:277-82.

26. Whitehead AL, Julious SA, Cooper CL, Campbell MJ. Estimating the sample size for a pilot randomised trial to minimise the overall trial sample size for the external pilot and main trial for a continuous outcome variable. Stat Methods Med Res. 2016;25:1057-73.

27. Connell JT. A novel method to assess antihistamine and decongestant efficacy. Ann Allergy. 1979;42:278-85.

28. Roth RP, Cantekin EI, Bluestone CD, Welch RM, Cho YW. Nasal decongestant activity of pseudoephedrine. Ann Otol Rhinol Laryngol. 1977;86:235-42.

29. Hamilton LH, Chobanian SL, Cato A, Perkins JG. A study of sustained action pseudoephedrine in allergic rhinitis. Ann Allergy. 1982;48:87-92.

30. Berkowitz RB, McCafferty F, Lutz C, Bazelmans D, Godfrey P, Meeves $\mathrm{S}$, et al. Fexofenadine $\mathrm{HCl} 60 \mathrm{mg} /$ pseudoephedrine $\mathrm{HCl} 120 \mathrm{mg}$ has a 60 -minute onset of action in the treatment of seasonal allergic rhinitis symptoms, as assessed in an allergen exposure unit. Allergy Asthma Proc. 2004; $25: 335-43$. 
31. Enomoto T, Lu HQ, Yin M, Sakoda T, Dake Y, Enomoto K, et al. Evaluation of the efficacy and safety of olopatadine and fexofenadine compared with placebo in Japanese cedar pollinosis using an environmental exposure unit. J Investig Allergol Clin Immunol. 2009;19:299-305.

32. Eccles R. Nasal airflow in health and disease. Acta Otolaryngol. 2000;120:580-95.
33. Clarke RW, Jones AS. Nasal airflow sensation. Clin Otolaryngol Allied Sci. 1995;20:97-9.

34. Jones AS, Crosher R, Wight RG, Lancer JM, Beckingham E. The effect of local anaesthesia of the nasal vestibule on nasal sensation of airflow and nasal resistance. Clin Otolaryngol Allied Sci. 1987;12:461-4. 